\title{
Quantum Larmor radiation in conformally flat universe
}

\author{
Rampei Kimura, Gen Nakamura, Kazuhiro Yamamoto \\ Department of Physical Science, Hiroshima University, Higashi-Hiroshima 739-8526, Japan
}

\begin{abstract}
We investigate the quantum effect on the Larmor radiation from a moving charge in an expanding universe based on the framework of the scalar quantum electrodynamics (SQED). A theoretical formula for the radiation energy is derived at the lowest order of the perturbation theory with respect to the coupling constant of the SQED. We evaluate the radiation energy on the background universe so that the Minkowski spacetime transits to the Milne universe, in which the equation of motion for the mode function of the free complex scalar field can be exactly solved in an analytic way. Then, the result is compared with the WKB approach, in which the equation of motion of the mode function is constructed with the WKB approximation which is valid as long as the Compton wavelength is shorter than the Hubble horizon length. This demonstrates that the quantum effect on the Larmor radiation of the order $e^{2} \hbar$ is determined by a non-local integration in time depending on the background expansion. We also compare our result with a recent work by Higuchi and Walker [Phys. Rev. D80 105019 (2009)], which investigated the quantum correction to the Larmor radiation from a charged particle in a non-relativistic motion in a homogeneous electric field.
\end{abstract}

\section{INTRODUCTION}

Recently, the quantum electrodynamics (QED) processes in a strong field background attract interest of theoretical researchers as well as experimental researchers [1-3]. Especially, recent developments of strong laser technique might provide us with a chance to test the Schwinger effect, which causes a pair creation phenomenon in the strong laserelectric field [4]. On the other hand, some authors claimed that the Unruh effect [5, 6] might be detected by measuring the radiation from electron beam in a strong laser background [7, 8], but it seems to be necessary for more careful investigations for the definite conclusion of the relevance [9].

An analogy between the radiation process from a moving charge in an expanding universe and the classical Larmor radiation in the electro-magnetic theory has been investigated ([10], see also [11, 12]). The motion of a massive charged particle in an expanding background spacetime can be regarded as an accelerated motion because the physical momentum of the particle changes as the background spacetime expands or contracts. This leads to the Larmor radiation from the charge in an accelerated motion in the classical electro-magnetic theory. In Ref. [10], it was also demonstrated that the quantum effect affects the radiation energy using a model on a background universe with specific expansion, where the equation of motion of the mode function is solved in an analytic manner exactly.

In this paper, we focus our investigation on the quantum effect on the Larmor radiation from a moving charge in an expanding universe. Recently, Higuchi and Walker investigated the first order quantum effect of the Larmor radiation from a moving charge in a homogeneous electric field background [13]. In Ref. [13], the motion of the moving charge is limited to the non-relativistic case. A generalization of the work of [13] is presented in Ref. [9]. In the present paper, we apply the method developed in Ref. [9] to the radiation from a moving charge in an expanding universe.

This paper is organized as follows: In Sec. 2, we derive a general formula for the radiation energy of the quantum Larmor process in an expanding universe on the basis of the scalar QED. This formula, which is evaluated at the lowest order of the perturbation theory with respect to the coupling constant, is obtained by solving the equation of motion of the mode function of the complex scalar field in an expanding universe. In Sec. 3, we consider the complex scalar field on the background universe that the Minkowski spacetime transits to the Milne universe, on which the equation of motion of the mode function is exactly solved in an analytic manner. Then, the radiation energy is evaluated by performing an integration numerically. In Sec. 4, we adopt the WKB approach for the mode function, which is constructed in an expanded form with respect to $\hbar$. This approach yields the formula of the radiation energy in an expanded form with respect to $\hbar$ as well. The quantum effect of the order of $\hbar$ is determined by the non-local nature in time of the background expansion. The result is compared with the work [13]. Section 5 is devoted to the summary and conclusions. In Appendix A, we summarized useful formulas in our derivation of the radiation formula. Through out this paper, we set the speed of light $c=1$ and the metric convention $(+,-,-,-)$. 


\section{FORMULATION}

First, we derive the formula for the radiation energy from a moving charge in the spatially flat Friedmann-RobertsonWalker spacetime, whose line element is given by

$$
d s^{2}=a^{2}(\eta)\left[d \eta^{2}-\delta_{i j} d x^{i} d x^{j}\right]
$$

where $\eta$ is the conformal time and $a(\eta)$ is the scale factor. We consider the scalar QED action conformally coupled to the curvature

$$
S=\int d \eta d \mathbf{x} \sqrt{-g}\left[g^{\mu \nu}\left(\nabla_{\mu}-\frac{i e A_{\mu}}{\hbar}\right) \phi^{\dagger}\left(\nabla_{\nu}+\frac{i e A_{\nu}}{\hbar}\right) \phi-\left(\frac{m^{2}}{\hbar^{2}}-\xi R\right) \phi^{*} \phi-\frac{1}{4 \mu_{0}} F_{\mu \nu} F^{\mu \nu}\right]
$$

where $F_{\mu \nu}$ is the field strength, $\mu_{0}$ is the magnetic permeability of vacuum, and $\xi=1 / 6$. Introducing the conformally rescaled field $\Phi$,

$$
\phi=\frac{\Phi}{a(\eta)}
$$

we may rewrite the action as

$$
S=\int d \eta d \mathbf{x}\left[\left(D_{\mu} \Phi\right)^{\dagger} D^{\mu} \Phi-\frac{m^{2} a^{2}(\eta)}{\hbar^{2}} \Phi^{\dagger} \Phi-\frac{1}{4} F_{\mu \nu} F^{\mu \nu}\right]
$$

where $D_{\mu} \equiv \partial_{\mu}+i e A_{\mu} / \hbar$. It might be worthwhile to note that the equivalence between Eqs. (2) and (4) will break down at one-loop order [1]. However, this does not affect our result because our investigation is at tree level.

We adopt the in-in formalism [14] to evaluate the radiation energy, in which the expectation value of the Heisenberg operator $Q$ at the time $\eta$ is given by the following form,

$$
\langle Q(\eta)\rangle=\sum_{N=0}^{\infty} \frac{i^{N}}{\hbar^{N}} \int_{-\infty}^{\eta} d \eta_{N} \ldots \int_{-\infty}^{\eta_{2}} d \eta_{1}\left\langle\left[H_{I}\left(\eta_{1}\right), \ldots\left[H_{I}\left(\eta_{N}\right), Q(\eta)\right] \ldots\right]\right\rangle
$$

where $H_{I}$ denotes the interaction Hamiltonian operator,

$$
H_{I}(\eta)=-\frac{i e}{\hbar} \int d^{3} \mathbf{x} A^{\mu}(\eta, \mathbf{x})\left[\partial_{\mu} \Phi^{\dagger}(\eta, \mathbf{x}) \Phi(\eta, \mathbf{x})-\Phi^{\dagger}(\eta, \mathbf{x}) \partial_{\mu} \Phi(\eta, \mathbf{x})\right]
$$

and the operators in the right hand side of Eq. (5) are those of the interaction picture. The free field of the electromagnetic field quantized in a finite box is

$$
A_{\mu}=\sqrt{\frac{\mu_{0} \hbar}{V}} \sum_{\lambda=1,2} \sum_{\mathbf{k}} \sqrt{\frac{1}{2 k}} \epsilon_{\mu}^{\lambda}\left(a_{\mathbf{k}}^{\lambda} e^{-i k \eta}+a_{-\mathbf{k}}^{\lambda \dagger} e^{i k \eta}\right) e^{i \mathbf{k} \cdot \mathbf{x}}
$$

where $\epsilon_{\mu}^{\lambda}$ denotes the polarization tensor, $\lambda$ denotes the direction of polarization, $V$ is the volume of the box, $a_{\mathbf{k}}^{\lambda \dagger}$ and $a_{\mathbf{k}}^{\lambda}$ are the creation and annihilation operator, respectively, which satisfy the following commutation relation,

$$
\left[a_{\mathbf{k}}^{\lambda}, a_{\mathbf{k}^{\prime}}^{\dagger \lambda^{\prime}}\right]=\delta_{\lambda \lambda^{\prime}} \delta_{\mathbf{k}, \mathbf{k}^{\prime}}
$$

In the similar way, the quantized complex scalar field is given by

$$
\Phi(x)=\sum_{\mathbf{p}} \sqrt{\frac{\hbar}{V}} e^{i \mathbf{p} \cdot \mathbf{x} / \hbar}\left(\varphi_{\mathbf{p}}(\eta) b_{\mathbf{p}}+\varphi_{\mathbf{p}}^{*}(\eta) c_{-\mathbf{p}}^{\dagger}\right)
$$

where the mode function $\varphi_{\mathbf{p}}(\eta)$ obeys the equation of motion,

$$
\left(\frac{\partial^{2}}{\partial \eta^{2}}+\frac{\mathbf{p}^{2}+m^{2} a^{2}(\eta)}{\hbar^{2}}\right) \varphi_{\mathbf{p}}(\eta)=0
$$




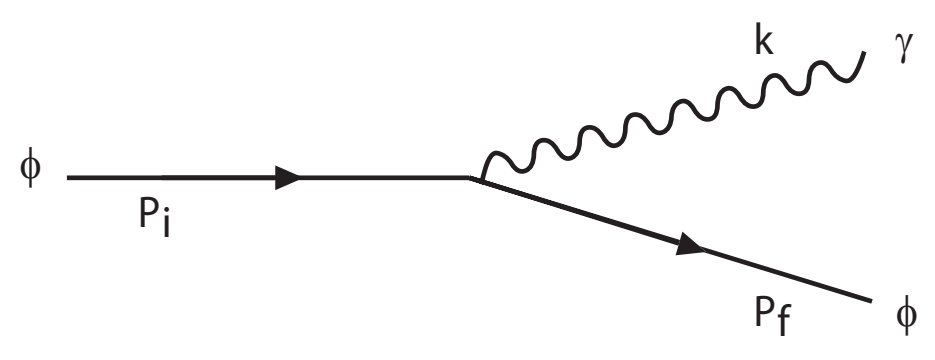

FIG. 1: Feynman Diagram for the process.

with the normalization condition $\dot{\varphi}_{\mathbf{p}}^{*} \varphi_{\mathbf{p}}-\dot{\varphi}_{\mathbf{p}} \varphi_{\mathbf{p}}=i$, and the creation and annihilation operators satisfy the commutation relation,

$$
\left[b_{\mathbf{p}}, b_{\mathbf{p}^{\prime}}^{\dagger}\right]=\delta_{\mathbf{p}, \mathbf{p}^{\prime}}, \quad\left[c_{\mathbf{p}}, c_{\mathbf{p}^{\prime}}^{\dagger}\right]=\delta_{\mathbf{p}, \mathbf{p}^{\prime}}, \quad[\mathrm{OTHER}]=0 .
$$

Choosing the Heisenberg operator $Q$ as the number operator $a_{\mathbf{k}}^{\lambda \dagger} a_{\mathbf{k}}^{\lambda}$, we calculate the total radiation energy as follows,

$$
E=\int d^{3} \mathbf{k} \hbar k \sum_{\lambda}\left\langle a_{\mathbf{k}}^{\lambda \dagger} a_{\mathbf{k}}^{\lambda}\right\rangle=-\int d^{3} \mathbf{k} \hbar k \sum_{\lambda} \hbar^{-2} \int_{-\infty}^{t} d t_{2} \int_{-\infty}^{t_{2}} d t_{1}\left\langle\left[H_{I}\left(t_{1}\right),\left[H_{I}\left(t_{2}\right), a_{\mathbf{k}}^{\lambda \dagger I} a_{\mathbf{k}}^{\lambda I}\right]\right]\right\rangle,
$$

where we only considered the contribution up to the order of $e^{2}$. We set the initial state as,

$$
|i\rangle=|0\rangle_{a} \otimes|1\rangle_{b} \otimes|0\rangle_{\bar{b}}
$$

where the subscript $a$ means the photon number basis, $b$ is the charged particle number basis, and $\bar{b}$ is the anti-particle number basis.

We consider the lowest order contribution of the process so that one photon is emitted from the charged particle, as shown in Fig. 1. From Eq. (12), we have

$$
\begin{aligned}
E=-\frac{e^{2}}{\epsilon_{0}} \int \frac{d^{3} k}{(2 \pi)^{3}} & k\left\{\left|\int d \eta \frac{e^{i k \eta}}{\sqrt{2 k}}\left(\frac{\partial}{\partial \eta} \varphi_{\mathbf{p}_{f}}(\eta)^{*} \varphi_{\mathbf{p}_{i}}(\eta)-\varphi_{\mathbf{p}_{f}}^{*}(\eta) \frac{\partial}{\partial \eta} \varphi_{\mathbf{p}_{i}}(\eta)\right)\right|^{2}\right. \\
& \left.-\left|\int d \eta \frac{e^{i k \eta}}{\sqrt{2 k}}\left(\frac{i \mathbf{p}_{f}}{\hbar} \varphi_{\mathbf{p}_{f}}(\eta)^{*} \varphi_{\mathbf{p}_{i}}(\eta)+\varphi_{\mathbf{p}_{f}}^{*}(\eta) \frac{i \mathbf{p}_{i}}{\hbar} \varphi_{\mathbf{p}_{i}}(\eta)\right)\right|^{2}\right\}
\end{aligned}
$$

where $\mathbf{p}_{f}=\mathbf{p}_{i}-\hbar \mathbf{k}, \hbar \mathbf{k}$ is the photon momentum, and $\epsilon_{0}$ is the permittivity of vacuum, which is related to $\mu_{0}$ by $\epsilon_{0} \mu_{0}=1 / c^{2}=1$. Using the equation of motion (10), Eq. (14) leads to

$$
\begin{aligned}
E=-\frac{e^{2}}{\epsilon_{0}} \int \frac{d^{3} k}{(2 \pi)^{3}} & k\left\{\left|\int d \eta \frac{e^{i k \eta}}{\sqrt{2 k}} \frac{\hat{\mathbf{k}} \cdot\left(\mathbf{p}_{i}+\mathbf{p}_{f}\right)}{\hbar} \varphi_{\mathbf{p}_{f}}^{*}(\eta) \varphi_{\mathbf{p}_{i}}(\eta)\right|^{2}\right. \\
& \left.-\left|\int d \eta \frac{e^{i k \eta}}{\sqrt{2 k}} \frac{\mathbf{p}_{i}+\mathbf{p}_{f}}{\hbar} \varphi_{\mathbf{p}_{f}}^{*}(\eta) \varphi_{\mathbf{p}_{i}}(\eta)\right|^{2}\right\}
\end{aligned}
$$

where $\hat{\mathbf{k}}=\mathbf{k} /|\mathbf{k}|$. Furthermore, Eq. (15) reduces to (cf. [10])

$$
E=\frac{e^{2}}{2 \epsilon_{0}} \int \frac{d^{3} k}{(2 \pi)^{3}} \frac{4\left(\mathbf{p}_{i}^{2}-\left(\mathbf{p}_{i} \cdot \hat{\mathbf{k}}\right)^{2}\right)}{\hbar^{2}}\left|\int d \eta e^{i k \eta} \varphi_{\mathbf{p}_{f}}^{*}(\eta) \varphi_{\mathbf{p}_{i}}(\eta)\right|^{2}
$$

Note that this is the energy in the conformally rescaled spacetime, which is not the physical energy. We can read the physical radiation energy as $\mathcal{E}=E / a$.

\section{SOLVABLE MODEL IN MINKOWSKI-MILNE UNIVERSE}

In this section, we consider the universe whose scale factor is written in the form,

$$
a(\eta)=\sqrt{1+e^{2 \rho \eta}}
$$


where $\rho$ is the parameter to describe the expansion rate. This gives the Minkowski spacetime at $\eta \rightarrow-\infty$, and the Milne universe at $\eta \rightarrow+\infty$. The equation of motion of the mode function (10) is

$$
\left[\frac{d^{2}}{d \eta^{2}}+\frac{p^{2}+m^{2}\left(1+e^{2 \rho \eta}\right)}{\hbar^{2}}\right] \varphi_{p}(\eta)=0
$$

The solution is given by using the Bessel function,

$$
\varphi_{p}(\eta)=\sqrt{\frac{\pi}{2 \rho \sinh \pi \xi}} J_{-i \xi}\left(\bar{m} e^{\rho \eta}\right),
$$

where

$$
\xi=\sqrt{\frac{\mathbf{p}^{2}+m^{2}}{\rho^{2} \hbar^{2}}}, \quad \bar{m}=\frac{m}{\rho \hbar} .
$$

The radiation energy is given by the formula (16), then we focus on

$$
\begin{aligned}
\int_{-\infty}^{\infty} d \eta e^{i k \eta} \varphi_{\mathbf{p}_{f}}^{*}(\eta) \varphi_{\mathbf{p}_{i}}(\eta) & =\sqrt{\frac{\pi}{2 \rho \sinh \pi \xi_{f}}} \sqrt{\frac{\pi}{2 \rho \sinh \pi \xi_{i}}} \int_{-\infty}^{\infty} d \eta e^{i k \eta} J_{i \xi_{f}}\left(\bar{m} e^{\rho \eta}\right) J_{-i \xi_{i}}\left(\bar{m} e^{\rho \eta}\right), \\
& =\sqrt{\frac{\pi}{2 \rho \sinh \pi \xi_{f}}} \sqrt{\frac{\pi}{2 \rho \sinh \pi \xi_{i}}} \frac{\bar{m}^{-i k / \rho}}{\rho} \int_{0}^{\infty} d z z^{i k / \rho-1} J_{i \xi_{f}}(z) J_{-i \xi_{i}}(z),
\end{aligned}
$$

where we defined

$$
\xi_{i}=\sqrt{\frac{\mathbf{p}_{i}^{2}+m^{2}}{\rho^{2} \hbar^{2}}}, \quad \xi_{f}=\sqrt{\frac{\mathbf{p}_{f}^{2}+m^{2}}{\rho^{2} \hbar^{2}}}=\sqrt{\frac{\mathbf{p}_{i}^{2}-2 \hbar k p_{i} \cos \theta+\hbar^{2} k^{2}+m^{2}}{\rho^{2} \hbar^{2}}} .
$$

We use the mathematical formula [15]

$$
\int_{0}^{\infty} d z z^{i k / \rho-1} J_{i \xi_{f}}(z) J_{-i \xi_{i}}(z)=\frac{2^{i \kappa-1} \Gamma(1-i \kappa) \Gamma\left(i\left(\xi_{f}-\xi_{i}+\kappa\right) / 2\right)}{\Gamma\left(i\left(\xi_{f}-\xi_{i}-\kappa\right) / 2+1\right) \Gamma\left(i\left(\xi_{f}+\xi_{i}-\kappa\right) / 2+1\right) \Gamma\left(i\left(-\xi_{f}+\xi_{i}-\kappa\right) / 2+1\right)},
$$

where we defined $\kappa=k / \rho$. Then, the radiation energy is

$$
\begin{aligned}
E=\frac{e^{2} p_{i}^{2}}{2 \pi \epsilon_{0} \rho^{4}} & \int_{0}^{\infty} d k k^{2} \int_{-1}^{1} d \cos \theta\left(1-\cos ^{2} \theta\right) \\
& \times \frac{1}{\sinh \pi \xi_{f}} \frac{1}{\sinh \pi \xi_{i}} \frac{\kappa}{\sinh \pi \kappa} \frac{1}{\left(\xi_{f}-\xi_{i}+\kappa\right) \sinh \pi\left(\xi_{f}-\xi_{i}+\kappa\right) / 2} \\
& \times \frac{\sinh \pi\left(\xi_{f}+\xi_{i}-\kappa\right) / 2}{\xi_{f}+\xi_{i}-\kappa} \frac{\sinh \pi\left(\xi_{f}+\xi_{i}+\kappa\right) / 2}{\xi_{f}+\xi_{i}+\kappa} \frac{\sinh \pi\left(\xi_{f}-\xi_{i}-\kappa\right) / 2}{\xi_{f}-\xi_{i}-\kappa} .
\end{aligned}
$$

The classical radiation energy corresponding to the Larmor radiation can be evaluated using Eq. (43) (see also Eq. (36) of Ref. [10]). In the case of the background universe with Eq. (17), we have

$$
E_{\mathrm{cl}}=\frac{e^{2} p_{i}^{2} \rho}{24 \pi \epsilon_{0} m^{2}} .
$$

Combining (23) and (24), we have

$$
\begin{aligned}
& \frac{E}{E_{\mathrm{cl}}}=12\left(\frac{m}{\rho \hbar}\right)^{2} \int_{0}^{\infty} d \kappa \kappa^{2} \int_{-1}^{1} d \cos \theta\left(1-\cos ^{2} \theta\right) \\
& \quad \times \frac{1}{\sinh \pi \xi_{f}} \frac{1}{\sinh \pi \xi_{i}} \frac{\kappa}{\sinh \pi \kappa} \frac{1}{\left(\xi_{f}-\xi_{i}+\kappa\right) \sinh \pi\left(\xi_{f}-\xi_{i}+\kappa\right) / 2} \\
& \times \frac{\sinh \pi\left(\xi_{f}+\xi_{i}-\kappa\right) / 2}{\xi_{f}+\xi_{i}-\kappa} \frac{\sinh \pi\left(\xi_{f}+\xi_{i}+\kappa\right) / 2}{\xi_{f}+\xi_{i}+\kappa} \frac{\sinh \pi\left(\xi_{f}-\xi_{i}-\kappa\right) / 2}{\xi_{f}-\xi_{i}-\kappa} .
\end{aligned}
$$

We performed the integration of Eq. (25) numerically. Figure 2 plots $E / E_{\mathrm{cl}}$ as a function of $m / \rho \hbar$ with fixing $p_{i} / m=0.1$ (black solid curve), $p_{i} / m=1$ (red dashed curve), and $p_{i} / m=2$ (blue dotted curve). In the limit $m / \rho \hbar \gg 1$, one can see that $E / E_{\mathrm{cl}}$ approaches to 1 . On the other hand, in the limit $m / \rho \hbar \ll 1, E / E_{\mathrm{cl}}$ approaches to 0 . Complimentary to this figure, Figure 3 shows the contour of $E / E_{\mathrm{cl}}$ on the plane $p_{i} / m$ and $m / \rho \hbar$. For the region $m / \rho \hbar \gg 1, E / E_{\mathrm{cl}}$ approaches to 1 , while for the region $m / \rho \hbar \ll 1, E / E_{\mathrm{cl}}$ approaches to 0 . These features are the same as those in the previous paper [10], though a different background universe was considered there. 


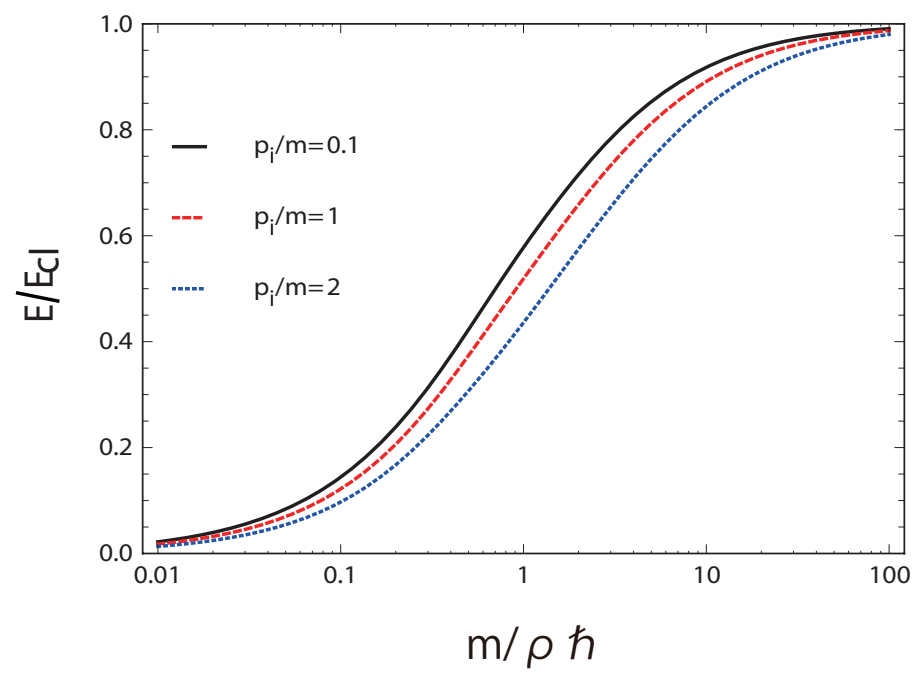

FIG. 2: The ratio of the total radiation energy to the classical WKB formula, $E / E_{\mathrm{cl}}$, as function of $m / \rho \hbar$ with fixing $p_{i} / m=0.1$ (black solid curve), $p_{i} / m=1$ (red dashed curve), and $p_{i} / m=2$ (blue dotted curve).

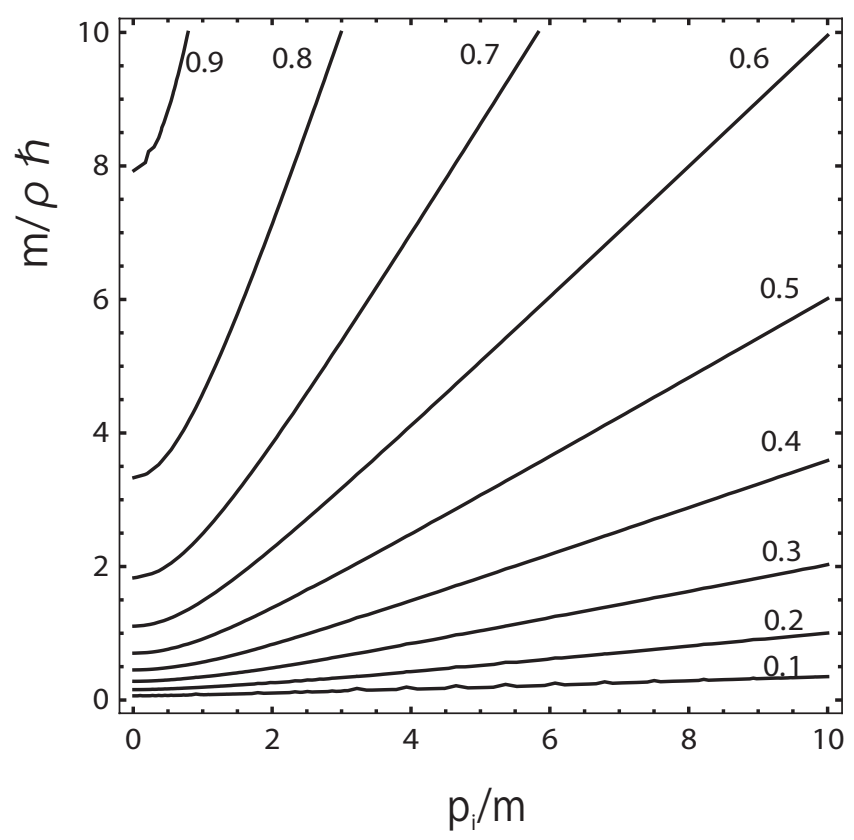

FIG. 3: The deviation from the classical WKB formula. Contour of $E / E_{\mathrm{cl}}$ on the $p_{i} / m$ and $m / \rho \hbar$ plane.

\section{WKB APPROACH}

In the previous section, we investigated the radiation energy in the case when the mode function is exactly solved in the analytic manner. From the result of the exactly solvable model in the previous section as well as the previous paper [10], the quantum correction reduces the total radiation energy compared with the classical Larmor formula. In this section, we consider the origin of the quantum effect in the Larmor radiation. To this end, we adopt the WKB approach. Our computation is a generalization of the work by Higuchi and Walker [13], in which the quantum effect of the Larmor radiation on an electric-field background is investigated (cf. [9]).

The mode function with the WKB approximation is

$$
\varphi_{\mathbf{p}}(\eta)=\frac{1}{\sqrt{2 \Omega_{\mathbf{p}}}} \exp \left[-i \int^{\eta} \Omega_{\mathbf{p}}\left(\eta^{\prime}\right) d \eta^{\prime}\right]
$$


with

$$
\Omega_{\mathbf{p}}(\eta)=\frac{\sqrt{\mathbf{p}^{2}+m^{2} a^{2}(\eta)}}{\hbar} .
$$

Substituting the WKB solution (26) into Eq. (16), we have

$$
\begin{aligned}
E=-\frac{e^{2}}{2 \epsilon_{0}} \frac{1}{2^{2}} & \int \frac{d^{3} k}{(2 \pi)^{3}}\left\{\left|\int d \eta \frac{\hat{\mathbf{k}} \cdot\left(2 \mathbf{p}_{i}-\hbar \mathbf{k}\right)}{\hbar \sqrt{\Omega_{\mathbf{p}_{i}}} \sqrt{\Omega_{\mathbf{p}_{f}}}} \exp \left[i k \eta+i \int^{\eta}\left(\Omega_{\mathbf{p}_{f}}-\Omega_{\mathbf{p}_{i}}\right) d \eta^{\prime}\right]\right|^{2}\right. \\
& \left.-\left|\int d \eta \frac{2 \mathbf{p}_{i}-\hbar \mathbf{k}}{\hbar \sqrt{\Omega_{\mathbf{p}_{i}}} \sqrt{\Omega_{\mathbf{p}_{f}}}} \exp \left[i k \eta+i \int^{\eta}\left(\Omega_{\mathbf{p}_{f}}-\Omega_{\mathbf{p}_{i}}\right) d \eta^{\prime}\right]\right|^{2}\right\}
\end{aligned}
$$

with

$$
\begin{aligned}
& \Omega_{\mathbf{p}_{i}}(\eta)=\sqrt{\mathbf{p}_{i}^{2}+m^{2} a^{2}(\eta)} / \hbar \\
& \Omega_{\mathbf{p}_{f}}(\eta)=\sqrt{\left(\mathbf{p}_{i}-\hbar \mathbf{k}\right)^{2}+m^{2} a^{2}(\eta)} / \hbar .
\end{aligned}
$$

We derive the expression for the radiation energy in the form expanded as a power series with respect to $\hbar$. Using the approximation,

$$
\begin{aligned}
& \Omega_{\mathbf{p}_{f}}-\Omega_{\mathbf{p}_{i}} \simeq-\frac{\mathbf{k} \cdot \mathbf{p}_{i}}{\sqrt{\mathbf{p}_{i}^{2}+m^{2} a^{2}(\eta)}}+\frac{\hbar}{2}\left(\frac{k^{2}}{\sqrt{\mathbf{p}_{i}^{2}+m^{2} a^{2}(\eta)}}-\frac{\left(\mathbf{k} \cdot \mathbf{p}_{i}\right)^{2}}{\sqrt{\mathbf{p}_{i}^{2}+m^{2} a^{2}(\eta)}}\right), \\
& \frac{1}{\hbar \sqrt{\Omega_{\mathbf{p}_{i}}} \sqrt{\Omega_{\mathbf{p}_{f}}}} \simeq \frac{1}{\sqrt{\mathbf{p}_{i}^{2}+m^{2} a^{2}(\eta)}}\left\{1+\frac{\hbar}{2} \frac{\mathbf{k} \cdot \mathbf{p}_{i}}{\mathbf{p}_{i}^{2}+m^{2} a^{2}(\eta)}\right\},
\end{aligned}
$$

we have the expression of the radiation energy up to the order of $\hbar$,

$$
\begin{aligned}
E= & -\frac{e^{2}}{2 \epsilon_{0}} \int \frac{d^{3} k}{(2 \pi)^{3}} \int d \xi \int d \xi^{\prime}\left\{\left(\hat{\mathbf{k}} \cdot \frac{d \mathbf{x}}{d \xi}\right)\left(\hat{\mathbf{k}} \cdot \frac{d \mathbf{x}^{\prime}}{d \xi^{\prime}}\right)-\frac{d \mathbf{x}}{d \xi} \cdot \frac{d \mathbf{x}^{\prime}}{d \xi^{\prime}}\right\} e^{i k \xi-i k \xi^{\prime}} \\
& \times\left[1+\frac{\hbar k}{2} \frac{\hat{\mathbf{k}} \cdot \mathbf{p}_{i}}{\mathbf{p}_{i}^{2}+m^{2} a^{2}(\eta)}+\frac{\hbar k}{2} \frac{\mathbf{k} \cdot \mathbf{p}_{i}}{\mathbf{p}_{i}^{2}+m^{2} a^{2}\left(\eta^{\prime}\right)}\right. \\
& \left.+\frac{i \hbar}{2} \int_{\eta^{\prime}}^{\eta}\left(\frac{k^{2}}{\sqrt{\mathbf{p}_{i}^{2}+m^{2} a^{2}\left(\eta^{\prime \prime}\right)}}-\frac{k^{2}\left(\hat{\mathbf{k}} \cdot \mathbf{p}_{i}\right)^{2}}{\sqrt{\mathbf{p}_{i}^{2}+m^{2} a^{2}\left(\eta^{\prime \prime}\right)}}\right) d \eta^{\prime \prime}+\mathcal{O}\left(\hbar^{2}\right)\right],
\end{aligned}
$$

where we introduced the new variable $\xi$ instead of $\eta$

$$
\xi=\eta-\int^{\eta} \frac{\hat{\mathbf{k}} \cdot \mathbf{p}_{i}}{\sqrt{\mathbf{p}_{i}^{2}+m^{2} a^{2}\left(\eta^{\prime}\right)}} d \eta^{\prime} .
$$

Furthermore, we introduce the notation,

$$
\begin{aligned}
& \frac{d \mathbf{x}^{i}}{d \tau}=\mathbf{p}_{i} \\
& \frac{d \eta}{d \tau}=\sqrt{\mathbf{p}_{i}^{2}+m^{2} a^{2}(\eta)}
\end{aligned}
$$

Then, Eq. (31) is rephrased as

$$
\begin{aligned}
E= & -\frac{e^{2}}{2 \epsilon_{0}} \int \frac{d^{3} k}{(2 \pi)^{3}} \int d \xi \int d \xi^{\prime}\left\{\left(\hat{\mathbf{k}} \cdot \frac{d \mathbf{x}}{d \xi}\right)\left(\hat{\mathbf{k}} \cdot \frac{d \mathbf{x}^{\prime}}{d \xi^{\prime}}\right)-\frac{d \mathbf{x}}{d \xi} \cdot \frac{d \mathbf{x}^{\prime}}{d \xi^{\prime}}\right\} e^{i k \xi-i k \xi^{\prime}} \\
& \times\left[1+\frac{\hbar k}{2}\left(\hat{\mathbf{k}} \cdot \frac{d \mathbf{x}}{d \eta} \frac{d \tau}{d \eta}+\hat{\mathbf{k}} \cdot \frac{d \mathbf{x}^{\prime}}{d \eta^{\prime}} \frac{d \tau^{\prime}}{d \eta^{\prime}}\right)+\frac{i \hbar k^{2}}{2} \int_{\tau\left(\xi^{\prime}\right)}^{\tau(\xi)} d \tau^{\prime \prime}\left(1-\left(\hat{\mathbf{k}} \cdot \frac{d \mathbf{x}^{\prime \prime}}{d \eta^{\prime \prime}}\right)^{2}\right)\right]
\end{aligned}
$$


We replace $k$ in Eq. (35) with the partial derivative with respect to $\xi$ or $\xi^{\prime}$ operating on $e^{i k \xi-i k \xi^{\prime}}$, which is the equivalent technique adopted in Ref. [13]. Partial integrations lead to

$$
\begin{aligned}
E^{(0)}= & -\frac{e^{2}}{2 \epsilon_{0}(2 \pi)^{3}} \int d \hat{\mathbf{k}} \int_{0}^{\infty} d k \int d \xi \int d \xi^{\prime} e^{i k\left(\xi-\xi^{\prime}\right)}\left(\left(\hat{\mathbf{k}} \cdot \frac{d^{2} \mathbf{x}}{d \xi^{2}}\right)\left(\hat{\mathbf{k}} \cdot \frac{d^{2} \mathbf{x}^{\prime}}{d \xi^{\prime 2}}\right)-\frac{d^{2} \mathbf{x}}{d \xi^{2}} \cdot \frac{d^{2} \mathbf{x}^{\prime}}{d \xi^{\prime 2}}\right) \\
E^{(1)}= & -\frac{e^{2}}{2 \epsilon_{0}(2 \pi)^{3}} \int d \hat{\mathbf{k}} \int_{0}^{\infty} d k \int d \xi \int d \xi^{\prime} e^{i k\left(\xi-\xi^{\prime}\right)} \\
& \times\left\{\frac{i \hbar}{4}\left(\frac{d}{d \xi}-\frac{d}{d \xi^{\prime}}\right) \frac{d}{d \xi} \frac{d}{d \xi^{\prime}}\left[\left(\hat{\mathbf{k}} \cdot \frac{d \mathbf{x}}{d \xi}\right)\left(\hat{\mathbf{k}} \cdot \frac{d \mathbf{x}^{\prime}}{d \xi^{\prime}}\right)-\frac{d \mathbf{x}}{d \xi} \cdot \frac{d \mathbf{x}^{\prime}}{d \xi^{\prime}}\right)\left(\hat{\mathbf{k}} \cdot \frac{d \mathbf{x}}{d \eta} \frac{d \tau}{d \eta}+\hat{\mathbf{k}} \cdot \frac{d \mathbf{x}^{\prime}}{d \eta^{\prime}} \frac{d \tau^{\prime}}{d \eta^{\prime}}\right)\right] \\
& +\frac{i \hbar}{2} \frac{d^{2}}{d \xi^{2}} \frac{d^{2}}{d \xi^{\prime 2}}\left[\left(\left(\hat{\mathbf{k}} \cdot \frac{d \mathbf{x}}{d \xi}\right)\left(\hat{\mathbf{k}} \cdot \frac{d \mathbf{x}^{\prime}}{d \xi^{\prime}}\right)-\frac{d \mathbf{x}}{d \xi} \cdot \frac{d \mathbf{x}^{\prime}}{d \xi^{\prime}}\right) \int_{\xi^{\prime}\left(\eta^{\prime}\right)}^{\xi(\eta)} d \xi^{\prime \prime} \frac{d \tau^{\prime \prime}}{d \xi^{\prime \prime}}\left(1-\left(\hat{\mathbf{k}} \cdot \frac{d \mathbf{x}^{\prime \prime}}{d \eta^{\prime \prime}}\right)\right]\right\}
\end{aligned}
$$

where $E^{(0)}$ and $E^{(1)}$ are the contribution of the order of $\hbar^{0}$ and $\hbar$, respectively. The expression of the order of $\hbar^{0}$, Eq. (36), gives the classical radiation energy formula [10, 11 (cf. [16 18]), while the expression of the order of $\hbar$, Eq. (37), gives the first order quantum effect in the radiation [9, 13]. We choose the coordinate $z$ to be parallel to $\mathbf{p}_{i}$, and the polar angle $\theta$ to be the angle between the $z$-axis and photon momentum vector $\mathbf{k}$. Then, we write

$$
\begin{aligned}
& v=\frac{d z}{d \eta}, \\
& \frac{d \xi}{d \eta}=(1-v \cos \theta), \\
& \frac{d^{2} z}{d \xi^{2}}=\frac{\dot{v}}{(1-v \cos \theta)^{3}},
\end{aligned}
$$

with which we have

$$
E^{(0)}=\frac{e^{2}}{8 \pi \epsilon_{0}} \int_{-1}^{1} d \cos \theta\left(1-\cos ^{2} \theta\right) \int d \xi\left(\frac{d^{2} z}{d \xi^{2}}\right)^{2}
$$

from Eq. (36). Here, the 'dot' denotes the differentiation with respect to $\eta$. Integration with respect to $\theta$ yields

$$
E^{(0)}=\frac{e^{2}}{6 \pi \epsilon_{0}} \int d \eta \frac{\dot{v}^{2}}{\left(1-v^{2}\right)^{3}}
$$

With the use of the relation $v=p_{i} / \sqrt{p_{i}^{2}+m^{2} a^{2}(\eta)}$, Eq. (42) reduces to

$$
E^{(0)}=\frac{e^{2} p_{i}^{2}}{6 \pi \epsilon_{0} m^{2}} \int d \eta \frac{\dot{a}^{2}}{a^{4}}
$$

This is the classical radiation formula in an expanding universe which can be regarded as the Larmor radiation [10].

Next, we consider the contribution of the order of $\hbar$, Eq. (37). Let us first consider the limit of the non-relativistic motion, $v \ll 1$, we can write the radiation energy in terms of the velocity and its time derivatives, and find the leading contribution (see Eq. (A3) in Appendix A),

$$
\begin{aligned}
E^{(1)}= & \frac{3 e^{2} \hbar}{4 \epsilon_{0}(2 \pi)^{2}} \int d \cos \theta \int d \eta \int d \eta^{\prime} \frac{1}{\eta-\eta^{\prime}} \\
& \times\left[\frac{1-\cos ^{2} \theta}{p_{i}}\left\{\left(\ddot{v} \dot{v}^{\prime} v^{\prime}-\ddot{v}^{\prime} \dot{v} v\right)+\dot{v}^{\prime}\left(3\left(\dot{v} v^{\prime}-\dot{v}^{\prime} v\right)-\left(\dot{v} v-\dot{v}^{\prime} v^{\prime}\right)\right) \cos \theta\right\}\right],
\end{aligned}
$$

where the 'dash' denotes the function of $\eta^{\prime}$, i.e., $v^{\prime}=v\left(\eta^{\prime}\right)$. Note that the latter terms of the right-hand-side of Eq. (44), which is in proportion to $\cos \theta$, give no contribution when integrated over $\theta$. In the non-relativistic case, we have

$$
\left|\frac{\ddot{v}}{\dot{v}^{2}}\right| \simeq \frac{m a}{p_{i}}\left|\frac{\ddot{a} a-2 \dot{a}^{2}}{\dot{a}^{2}}\right|
$$


This gives a rough estimation of the ratio of the first term ( $\left.\ddot{v} \dot{v}^{\prime} v^{\prime}-\ddot{v}^{\prime} \dot{v} v\right)$ in the right-hand-side of Eq. (44) to the latter terms in proportion to $\cos \theta$. Therefore, the first term is larger than the latter terms in the non-relativistic case, $m a / p_{i} \gg 1$.

Next, we consider the relativistic limit, $v \sim 1$. In an expanding universe, the condition of the relativistic motion is guaranteed as long as $m a(\eta) \lesssim p_{i}$. Hence, the particle motion becomes non-relativistic as the universe expands with (17), even though the motion is relativistic initially. In the model with (17), the particle motion is relativistic for

$$
\frac{m}{p_{i}} e^{\rho \eta} \ll 1
$$

On the other hand, the ratio of $\ddot{v}$ to $\dot{v}^{2}$ is written as

$$
\left|\frac{\ddot{v}}{\dot{v}^{2}}\right| \simeq \frac{p_{i}^{2}}{m^{2}}\left|\frac{\ddot{a} a+\dot{a}^{2}}{(\dot{a} a)^{2}}\right| \simeq \frac{p_{i}^{2}}{m^{2}} e^{-2 \rho \eta} .
$$

This means that $\left|\dot{v}^{2}\right| \ll|\ddot{v}|$ while the particle motion is relativistic. In this case, using expression (A5) in Appendix A, we have

$$
\begin{aligned}
E^{(1)} \simeq & \frac{3 e^{2} \hbar}{4 \epsilon_{0}(2 \pi)^{2}} \int d \cos \theta \int d \eta \int d \eta^{\prime} \frac{1}{\xi(\eta)-\xi\left(\eta^{\prime}\right)} \\
& \times\left[\frac{1-\cos ^{2} \theta}{p_{i}}\left\{\frac{\ddot{v}^{\prime}}{(1-v \cos \theta)^{3}\left(1-v^{\prime} \cos \theta\right)^{2}}-\frac{\ddot{v}^{\prime} \dot{v}}{(1-v \cos \theta)^{2}\left(1-v^{\prime} \cos \theta\right)^{3}}\right\}\right] .
\end{aligned}
$$

This expression is derived assuming the particle motion is relativistic all the time. Then, it is not justified when the particle motion changes from relativistic to non-relativistic as the universe expands, as for the model with (17) in Sec. 3.

\section{DISCUSSIONS}

Integrating the right-hand-side of Eq. (44) with respect to $\theta$, we obtain the quantum correction to the Larmor radiation of the order of $\hbar$,

$$
E^{(1)}=\frac{e^{2} \hbar}{4 \pi^{2} \epsilon_{0} p_{i}} \int d \eta \int d \eta^{\prime} \frac{\ddot{v} \dot{v}^{\prime} v^{\prime}-\ddot{v}^{\prime} \dot{v} v}{\eta-\eta^{\prime}} .
$$

This result should be compared with the result by Higuchi and Walker [13], which presented the quantum correction to the Larmor radiation of the order of $\hbar$ from a charged particle in an accelerated motion on a homogeneous electric field background,

$$
E_{H W}^{(1)}=\frac{e^{2} \hbar}{6 \pi^{2} \epsilon_{0} p_{i}} \int d t \int d t^{\prime} \frac{\ddot{v} \dot{v}^{\prime}-\ddot{v}^{\prime} \dot{v}}{t-t^{\prime}} .
$$

There is a difference between (49) and (50), i.e., the numerical coefficient and the dependence on the velocity and its derivatives. However, the quantum effect is non-local in time in both cases.

In the case when the velocity $v$ and its derivatives are approximated as

$$
\begin{aligned}
& v=\frac{p_{i}}{\sqrt{p_{i}^{2}+m^{2} a^{2}(\eta)}} \simeq \frac{p_{i}}{m a(\eta)}, \\
& \dot{v} \simeq-\frac{p_{i}}{m} \frac{\dot{a}}{a^{2}}, \\
& \ddot{v} \simeq-\frac{p_{i}}{m} \frac{\ddot{a} a-2 \dot{a}^{2}}{a^{3}},
\end{aligned}
$$

Eq. (49) yields

$$
E^{(1)}=\frac{e^{2} \hbar}{4 \pi^{2} \epsilon_{0} m} \frac{p_{i}^{2}}{m^{2}} \int d \eta \int d \eta^{\prime} \frac{1}{\eta-\eta^{\prime}}\left(\frac{\left(\ddot{a} a-2 \dot{a}^{2}\right) \dot{a}^{\prime}-\left(\ddot{a}^{\prime} a^{\prime}-2 \dot{a}^{\prime 2}\right) \dot{a}}{a^{3} a^{\prime 3}}\right)
$$

where $a=a(\eta)$ and $a^{\prime}=a\left(\eta^{\prime}\right)$. 


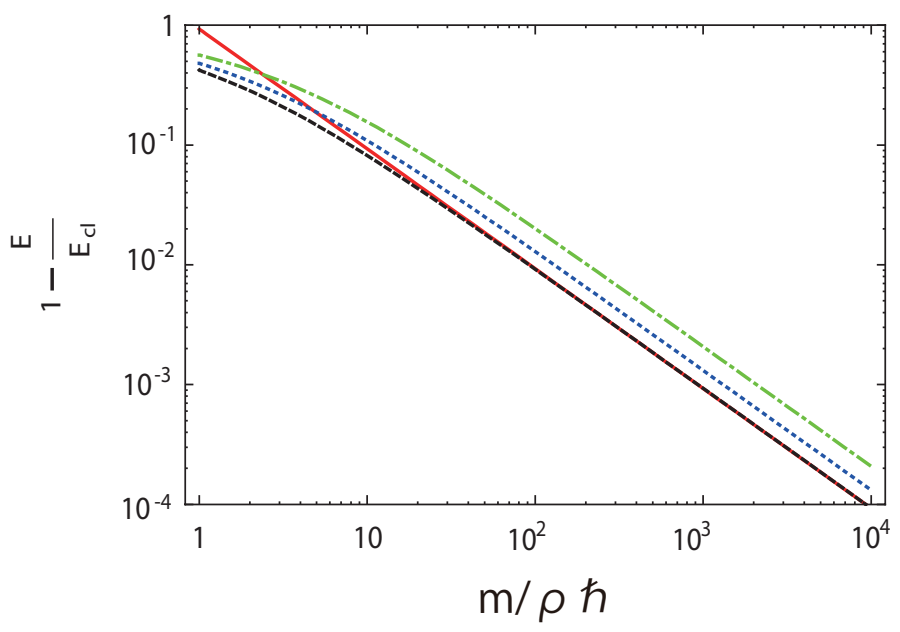

FIG. 4: Comparison of the deviation of the classical radiation from the total radiation. The solid (red) curve is $-E^{(1)} / E_{\mathrm{cl}}$ as a function of $m / \rho \hbar$, while the other curves are $-\left(E-E_{\mathrm{cl}}\right) / E_{\mathrm{cl}}$ with fixing $p_{i} / m=0.01$ (black dashed curve), $p_{i} / m=1$ (blue dotted curve), and $p_{i} / m=2$ (green dash-dotted curve), where $E^{(1)}$ is computed using Eq. (54).

Figure 4 shows the deviation of the total Larmor radiation from the classical Larmor radiation from a moving charge in the universe considered in Sec. 3, as a function of $m / \rho \hbar$. The curves compare the case when the radiation is computed with the exact mode function of Sec. 3 and the case when the radiation is computed with the WKB approaches up to the order of $\hbar$. The (black) dashed curve, the (blue) dotted curve, and the (green) dash-dotted curve are the results with the exact mode function with $p_{i} / m=0.01,1$, and 2 , respectively. The solid (red) curve is from the WKB mode function, Eq. (54). For the region with $p_{i} / m \ll 1$ and $m / \rho \hbar \gg 1$, the quantum correction can be well explained by the WKB approximate formula, Eq. (54). The condition $p_{i} / m \ll 1$ comes from the fact that Eq. (54) is derived in the non-relativistic approximation. The deviation of the results with $p_{i} / m=1$ and 2 from Eq. (54) comes from the fact that the particle motion is relativistic initially. However, the deviation is not significant, which may indicate that the fraction of the radiation from the particle in the non-relativistic regime is not small.

Finally, we show that the condition $m / \rho \hbar \gg 1$ is the necessary condition for the WKB approximation in an explicit manner. The WKB approximation holds under the condition [19],

$$
\frac{1}{2 \Omega^{2}}\left|\frac{\ddot{\Omega}}{\Omega}-\frac{3}{2} \frac{\dot{\Omega}^{2}}{\Omega^{2}}\right| \ll 1 .
$$

In the universe with the scale factor (17), the condition (55) is written as

$$
\left(\frac{\hbar \rho}{m}\right)^{2}\left|\frac{\left(a^{2}-1\right)\left(a^{2}-5-4 p_{i}^{2} / m^{2}\right)}{4\left(a^{2}+p_{i}^{2} / m^{2}\right)^{3}}\right| \ll 1
$$

Hence, the use of the WKB approximation is guaranteed when $m / \rho \hbar \gg 1$ is satisfied. This condition is interpreted as follows [10]. In the universe with (17), the ratio of the Compton wavelength to the Hubble horizon is

$$
\frac{\hbar \rho}{m} \frac{a^{2}-1}{a^{3}}
$$

When the condition $m / \rho \hbar \gg 1$ is satisfied, the ratio (57) is much less than unity. Thus, the condition for the WKB approximation can be regarded as the condition that the Compton wavelength is much shorter than the Hubble horizon.

\section{CONCLUSIONS}

In this paper, we investigated the quantum effect of the Larmor radiation from a moving charge in an expanding universe based on the framework of the perturbative approach of the scalar QED theory. We first derived the general formula for the quantum radiation energy, which is expressed by the mode function of the free complex scalar field. 
Assuming the background universe that the Minkowski spacetime transits to the Milne universe, in which the mode function is exactly solved in the analytic manner, we demonstrated how the total radiation energy deviates from the classical Larmor radiation formula. This gives us a hint to understand the quantum effect on the Larmor radiation.

In the latter part of the paper, we investigated the quantum effect of the Larmor radiation with the use of the WKB approximation in constructing the mode function. In the WKB approach, which is valid as long as the Compton wavelength is shorter than the Hubble horizon length, we investigated the quantum effect of the Larmor radiation by evaluating the contribution of the order of $\hbar$ of the radiation energy with the approximate mode function. The radiation of the order of $\hbar$ well explains the deviation of the total radiation with the exact approach from the classical Larmor formula in Sec. 3. The radiation of the order of $\hbar$ is determined by the non-local integration in time depending the behavior of the expansion of the background universe, as in the case of a homogeneous electric field background $[9$, 13]. However, the first order quantum correction to the Larmor radiation in the conformally flat universe is not the same as that on the homogeneous electric field background.

Acknowledgements K.Y. thanks A. Higuchi, H. Nomura and M. Sasaki for useful communication when he initiated the study of the present paper. He also thanks Y. Nambu for useful discussions. We are grateful for anonymous referee for useful comments. This work was supported by Japan Society for Promotion of Science (JSPS) Grants-inAid for Scientific Research (Nos. 21540270, 21244033). This work is also supported by JSPS Core-to-Core Program "International Research Network for Dark Energy". All the numerical computation presented in this paper were performed with the help of the package MATHEMATICA version 7.

\section{Appendix A: useful formulas}

In this Appendix, we summarize useful formulas in deriving the radiation formula in the present paper. Using Eqs. (33), (34), and (38)-(40), we have

$$
\begin{gathered}
\frac{d^{3} z}{d \xi^{3}}=\frac{\ddot{v}}{(1-v \cos \theta)^{4}}+\frac{3 \dot{v}^{2} \cos \theta}{(1-v \cos \theta)^{5}}, \\
\frac{d \tau}{d \eta}=\frac{1}{\sqrt{\mathbf{p}_{i}^{2}+m^{2} a^{2}(\eta)}}, \quad \frac{d}{d \xi}\left(v \frac{d \tau}{d \eta}\right)=\frac{2 \dot{v} v}{p_{i}(1-v \cos \theta)}, \quad \frac{d^{2}}{d \xi^{2}}\left(v \frac{d \tau}{d \eta}\right)=\frac{2 \dot{v}^{2}}{p_{i}(1-v \cos \theta)^{3}}+\frac{2 \ddot{v} v}{p_{i}(1-v \cos \theta)^{2}}, \\
\frac{d}{d \xi}\left(v \frac{d \tau}{d \eta}\right)=\frac{1}{1-v \cos \theta}\left(\frac{\dot{v}}{\sqrt{\mathbf{p}_{i}^{2}+m^{2} a^{2}(\eta)}}-\frac{v m^{2} a(\eta) \dot{a}(\eta)}{\sqrt{\mathbf{p}_{i}^{2}+m^{2} a^{2}(\eta)}}\right), \\
\frac{d^{2}}{d \xi^{2}}\left(v \frac{d \tau}{d \eta}\right)=\frac{\dot{v} \cos \theta}{(1-v \cos \theta)^{3}}\left(\frac { \dot { v } } { { \sqrt { \mathbf { p } _ { i } ^ { 2 } + m ^ { 2 } a ^ { 2 } ( \eta ) } } ^ { ( 1 - \frac { m ^ { 2 } a ( \eta ) \dot { a } ( \eta ) } { \mathbf { p } _ { i } ^ { 2 } + m ^ { 2 } a ^ { 2 } ( \eta ) } } ) + \frac { 1 } { ( 1 - v \operatorname { c o s } \theta ) ^ { 2 } } } \left(\frac{\ddot{v}}{\sqrt{\mathbf{p}_{i}^{2}+m^{2} a^{2}(\eta)}}\right.\right. \\
\left.-\frac{2 \dot{v} m^{2} a(\eta) \dot{a}(\eta)+v m^{2} \dot{a}(\eta) \dot{a}(\eta)+v m^{2} a(\eta) \ddot{a}(\eta)}{{\sqrt{\mathbf{p}_{i}^{2}+m^{2} a^{2}(\eta)}}^{3}}+\frac{3 v\left(m^{2} a(\eta) \dot{a}(\eta)\right)^{2}}{{\sqrt{\mathbf{p}_{i}^{2}+m^{2} a^{2}(\eta)}}^{5}}\right),
\end{gathered}
$$


where the dot denotes the differentiation with respect to the conformal time $\eta$. Using these relations and $\hat{\mathbf{k}} \cdot d \mathbf{x} / d \eta=$ $\cos \theta d z / d \eta=v \cos \theta$, it is straightforward to derive

$$
\begin{aligned}
& \left(\frac{d}{d \xi}-\frac{d}{d \xi^{\prime}}\right) \frac{d}{d \xi} \frac{d}{d \xi^{\prime}}\left[\left(\left(\hat{\mathbf{k}} \cdot \frac{d \mathbf{x}}{d \xi}\right)\left(\hat{\mathbf{k}} \cdot \frac{d \mathbf{x}^{\prime}}{d \xi^{\prime}}\right)-\frac{d \mathbf{x}}{d \xi} \cdot \frac{d \mathbf{x}^{\prime}}{d \xi^{\prime}}\right)\left(\hat{\mathbf{k}} \cdot \frac{d \mathbf{x}}{d \eta} \frac{d \tau}{d \eta}+\hat{\mathbf{k}} \cdot \frac{d \mathbf{x}^{\prime}}{d \eta^{\prime}} \frac{d \tau^{\prime}}{d \eta^{\prime}}\right)\right] \\
& =\left(\cos ^{2} \theta-1\right) \cos \theta\left\{\left[\left(\frac{\ddot{v}}{(1-v \cos \theta)^{4}}+\frac{3 \dot{v}^{2} \cos \theta}{(1-v \cos \theta)^{5}}\right)\left(\frac{\dot{v}^{\prime}}{\left(1-v^{\prime} \cos \theta\right)^{3}}\right)\right.\right. \\
& \left.-\left(\frac{\dot{v}}{(1-v \cos \theta)^{3}}\right)\left(\frac{\ddot{v}^{\prime}}{\left(1-v^{\prime} \cos \theta\right)^{4}}+\frac{3 \dot{v}^{\prime 2} \cos \theta}{\left(1-v^{\prime} \cos \theta\right)^{5}}\right)\right]\left(\frac{v^{2}+v^{\prime 2}}{p_{i}}\right) \\
& +2\left(\frac{\dot{v}}{(1-v \cos \theta)^{3}}\right)\left(\frac{\dot{v}^{\prime}}{\left(1-v^{\prime} \cos \theta\right)^{3}}\right)\left(\frac{2 v \dot{v}}{p_{i}(1-v \cos \theta)}-\frac{2 v^{\prime} \dot{v}^{\prime}}{p_{i}\left(1-v^{\prime} \cos \theta\right)}\right) \\
& +\left(\frac{v}{1-v \cos \theta}\right)\left(\frac{\dot{v}^{\prime}}{\left(1-v^{\prime} \cos \theta\right)^{3}}\right)\left(\frac{2 \dot{v}^{2}}{(1-v \cos \theta)^{3}}+\frac{2 v \ddot{v}}{(1-v \cos \theta)^{2}}\right) \frac{1}{p_{i}} \\
& -\left(\frac{\dot{v}}{(1-v \cos \theta)^{3}}\right)\left(\frac{v^{\prime}}{1-v^{\prime} \cos \theta}\right)\left(\frac{2 \dot{v}^{\prime 2}}{\left(1-v^{\prime} \cos \theta\right)^{3}}+\frac{2 v^{\prime} \ddot{v}^{\prime}}{\left(1-v^{\prime} \cos \theta\right)^{2}}\right) \frac{1}{p_{i}} \\
& +\left(\frac{\ddot{v}}{(1-v \cos \theta)^{4}}+\frac{3 \dot{v}^{2} \cos \theta}{(1-v \cos \theta)^{5}}\right)\left(\frac{v^{\prime}}{\left(1-v^{\prime} \cos \theta\right)}\right) \frac{2 v^{\prime} \dot{v}^{\prime}}{p_{i}\left(1-v^{\prime} \cos \theta\right)} \\
& \left.-\left(\frac{v}{(1-v \cos \theta)}\right)\left(\frac{\ddot{v}^{\prime}}{\left(1-v^{\prime} \cos \theta\right)^{4}}+\frac{3 \dot{v}^{\prime 2} \cos \theta}{\left(1-v^{\prime} \cos \theta\right)^{5}}\right) \frac{2 v \dot{v}}{p_{i}(1-v \cos \theta)}\right\},
\end{aligned}
$$

and

$$
\begin{aligned}
\frac{d^{2}}{d \xi^{2}} & \frac{d^{2}}{d \xi^{\prime 2}}\left[\left(\left(\hat{\mathbf{k}} \cdot \frac{d \mathbf{x}}{d \xi}\right)\left(\hat{\mathbf{k}} \cdot \frac{d \mathbf{x}^{\prime}}{d \xi^{\prime}}\right)-\frac{d \mathbf{x}}{d \xi} \cdot \frac{d \mathbf{x}^{\prime}}{d \xi^{\prime}}\right) \int_{\xi^{\prime}\left(\eta^{\prime}\right)}^{\xi(\eta)} d \xi^{\prime \prime} \frac{d \tau^{\prime \prime}}{d \xi^{\prime \prime}}\left(1-\left(\hat{\mathbf{k}} \cdot \frac{d \mathbf{x}^{\prime \prime}}{d \eta^{\prime \prime}}\right)^{2}\right)\right] \\
& =\left(\cos ^{2} \theta-1\right)\left\{\left(\frac{d^{3} z}{d \xi^{3}} \frac{d^{3} z^{\prime}}{d \xi^{\prime 3}}\right) \int_{\xi^{\prime}}^{\xi} d \xi^{\prime \prime} \frac{d \tau^{\prime \prime}}{d \xi^{\prime \prime}}\left(1-\cos ^{2} \theta\left(\frac{d z^{\prime \prime}}{d \eta^{\prime \prime}}\right)^{2}\right)\right. \\
& -2\left(\frac{\ddot{v}}{(1-v \cos \theta)^{4}}+\frac{3 \dot{v}^{2} \cos \theta}{(1-v \cos \theta)^{5}}\right)\left(\frac{\dot{v}^{\prime}}{\left(1-v^{\prime} \cos \theta\right)^{3}}\right) \frac{v^{\prime}\left(1+v^{\prime} \cos \theta\right)}{p_{i}} \\
& +2\left(\frac{\dot{v}}{(1-v \cos \theta)^{3}}\right)\left(\frac{\ddot{v}^{\prime}}{\left(1-\dot{v}^{\prime} \cos \theta\right)^{4}}+\frac{3 \dot{v}^{\prime 2} \cos \theta}{\left(1-v^{\prime} \cos \theta\right)^{5}}\right) \frac{v(1+v \cos \theta)}{p_{i}} \\
& -\left(\frac{\ddot{v}}{(1-v \cos \theta)^{4}}+\frac{3 \dot{v}^{2} \cos \theta}{(1-v \cos \theta)^{5}}\right)\left(\frac{v^{\prime}}{1-v^{\prime} \cos \theta}\right) \frac{\dot{v}^{\prime}\left(1+2 v^{\prime} \cos \theta\right)}{p_{i}\left(1-v^{\prime} \cos \theta\right)} \\
& \left.+\left(\frac{v}{1-v \cos \theta}\right)\left(\frac{\ddot{v}^{\prime}}{\left(1-v^{\prime} \cos \theta\right)^{4}}+\frac{3 \dot{v}^{\prime 2} \cos \theta}{\left(1-v^{\prime} \cos \theta\right)^{5}}\right) \frac{\dot{v}(1+2 v \cos \theta)}{p_{i}(1-v \cos \theta)}\right\}
\end{aligned}
$$

In the limit of the non-relativistic motion, $v \ll 1$, the above formulas give

$$
\begin{aligned}
& \frac{i \hbar}{4}\left\{\left(\frac{d}{d \xi}-\frac{d}{d \xi^{\prime}}\right) \frac{d}{d \xi} \frac{d}{d \xi^{\prime}}\left[\left(\left(\hat{\mathbf{k}} \cdot \frac{d \mathbf{x}}{d \xi}\right)\left(\hat{\mathbf{k}} \cdot \frac{d \mathbf{x}^{\prime}}{d \xi^{\prime}}\right)-\frac{d \mathbf{x}}{d \xi} \cdot \frac{d \mathbf{x}^{\prime}}{d \xi^{\prime}}\right)\left(\hat{\mathbf{k}} \cdot \frac{d \mathbf{x}}{d \eta} \frac{d \tau}{d \eta}+\hat{\mathbf{k}} \cdot \frac{d \mathbf{x}^{\prime}}{d \eta^{\prime}} \frac{d \tau^{\prime}}{d \eta^{\prime}}\right)\right]\right. \\
& \left.\quad+2 \frac{d^{2}}{d \xi^{2}} \frac{d^{2}}{d \xi^{\prime 2}}\left[\left(\left(\hat{\mathbf{k}} \cdot \frac{d \mathbf{x}}{d \xi}\right)\left(\hat{\mathbf{k}} \cdot \frac{d \mathbf{x}^{\prime}}{d \xi^{\prime}}\right)-\frac{d \mathbf{x}}{d \xi} \cdot \frac{d \mathbf{x}^{\prime}}{d \xi^{\prime}}\right) \int_{\xi^{\prime}\left(\eta^{\prime}\right)}^{\xi(\eta)} d \xi^{\prime \prime} \frac{d \tau^{\prime \prime}}{d \xi^{\prime \prime}}\left(1-\left(\hat{\mathbf{k}} \cdot \frac{d \mathbf{x}^{\prime \prime}}{d \eta^{\prime \prime}}\right)^{2}\right)\right]\right\} \\
& \simeq \frac{3 i \hbar}{2}\left[\frac{1-\cos ^{2} \theta}{p_{i}}\left\{\ddot{v} \dot{v}^{\prime} v^{\prime}-\ddot{v}^{\prime} \dot{v} v+\dot{v} \dot{v}^{\prime}\left(3\left(\dot{v} v^{\prime}-\dot{v}^{\prime} v\right)-\left(\dot{v} v-\dot{v}^{\prime} v^{\prime}\right)\right) \cos \theta\right\}\right] .
\end{aligned}
$$

Here, we omitted the contribution from the term,

$$
2\left(\cos ^{2} \theta-1\right)\left(\frac{d^{3} z}{d \xi^{3}} \frac{d^{3} z^{\prime}}{d \xi^{\prime 3}}\right) \int_{\xi^{\prime}}^{\xi} d \xi^{\prime \prime} \frac{d \tau^{\prime \prime}}{d \xi^{\prime \prime}}\left(1-\cos ^{2} \theta\left(\frac{d z^{\prime \prime}}{d \eta^{\prime \prime}}\right)^{2}\right)
$$

because its leading contribution to the radiation energy in Eq. (37) is zero when $\dot{v}$ is zero at the boundary $\eta \rightarrow \pm \infty$. 
In the limit of the relativistic motion, $v \sim v^{\prime} \sim 1$, we have

$$
\begin{aligned}
& \frac{i \hbar}{4}\left\{\left(\frac{d}{d \xi}-\frac{d}{d \xi^{\prime}}\right) \frac{d}{d \xi} \frac{d}{d \xi^{\prime}}\left[\left(\left(\hat{\mathbf{k}} \cdot \frac{d \mathbf{x}}{d \xi}\right)\left(\hat{\mathbf{k}} \cdot \frac{d \mathbf{x}^{\prime}}{d \xi^{\prime}}\right)-\frac{d \mathbf{x}}{d \xi} \cdot \frac{d \mathbf{x}^{\prime}}{d \xi^{\prime}}\right)\left(\hat{\mathbf{k}} \cdot \frac{d \mathbf{x}}{d \eta} \frac{d \tau}{d \eta}+\hat{\mathbf{k}} \cdot \frac{d \mathbf{x}^{\prime}}{d \eta^{\prime}} \frac{d \tau^{\prime}}{d \eta^{\prime}}\right)\right]\right. \\
& \left.\quad+2 \frac{d^{2}}{d \xi^{2}} \frac{d^{2}}{d \xi^{\prime 2}}\left[\left(\left(\hat{\mathbf{k}} \cdot \frac{d \mathbf{x}}{d \xi}\right)\left(\hat{\mathbf{k}} \cdot \frac{d \mathbf{x}^{\prime}}{d \xi^{\prime}}\right)-\frac{d \mathbf{x}}{d \xi} \cdot \frac{d \mathbf{x}^{\prime}}{d \xi^{\prime}}\right) \int_{\xi^{\prime}\left(\eta^{\prime}\right)}^{\xi(\eta)} d \xi^{\prime \prime} \frac{d \tau^{\prime \prime}}{d \xi^{\prime \prime}}\left(1-\left(\hat{\mathbf{k}} \cdot \frac{d \mathbf{x}^{\prime \prime}}{d \eta^{\prime \prime}}\right)^{2}\right)\right]\right\} \\
& \simeq \frac{i \hbar}{4} \frac{6\left(1-\cos ^{2} \theta\right)}{p_{i}(1-v \cos \theta)^{2}\left(1-v^{\prime} \cos \theta\right)^{2}}\left\{\frac{\ddot{v} \dot{v}^{\prime}}{(1-v \cos \theta)^{2}\left(1-v^{\prime} \cos \theta\right)}-\frac{\ddot{v}^{\prime} \dot{v}}{(1-v \cos \theta)\left(1-v^{\prime} \cos \theta\right)^{2}}\right\} .
\end{aligned}
$$

Here, again we omitted the contribution from the term (A4) because of the similar reason to that in the non-relativistic limit, as long as the particle motion is relativistic all the time. However, the omission is not justified when the particle motion changes from relativistic to non-relativistic as the universe expands.

[1] S. Iso, Y. Yamamoto, and S. Zhang, arXiv1011.4191

[2] K. Homma, D. Habs, and T. Tajima, arXiv:1006.4533.

[3] H. Gies, Eur. Phys. J. D55, (2009) 311.

[4] J. Schwinger, Phys. Rev. 82, 664 (1951)

[5] W. G. Unruh, Phys. Rev. D 14, 870 (1976)

[6] L. C. B. Crispino, A. Higuchi, and G. E. A. Matsas, Rev. Mod. Phys. 80, 787 (2008)

[7] P. Chen and T. Tajima, Phys. Rev. Lett. 83, 256 (1999)

[8] R. Schutzhold, G. Schaller, and D. Habs, Phys. Rev. Lett. 97, 121302 (2006)

[9] K. Yamamoto and G. Nakamura, Phys. Rev. D, in press, arXiv:1012.5182

[10] H. Nomura, M. Sasaki, and K. Yamamoto JCAP 0611013 (2006)

[11] A. Higuchi and P. J. Walker, Phys. Rev. D 79105023 (2009)

[12] T. Futamase, M. Hotta, H. Inoue, and M. Yamaguchi, Prog. Theor. Phys. 96, 113 (1996)

[13] A. Higuchi and P. J. Walker, Phys. Rev. D 80105019 (2009)

[14] S. Weinberg Phys. Rev. D 72043514 (2005)

[15] I. S. Gradshteyn and I. M. Ryzhik, Table of Integrals, Series, and Products (Academic Press, 1994)

[16] A. Higuchi and G. D. R. Martin, Found. Phys. 351149 (2005)

[17] A. Higuchi and G. D. R. Martin, Phys. Rev. D 73025019 (2006)

[18] A. Higuchi and G. D. R. Martin, Phys. Rev. D 74125002 (2006)

[19] N. D. Birrell and P. C. W. Davies, Quantum fields in curved space (Cambridge University Press ,1982) 